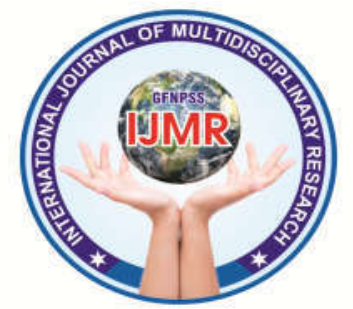

\title{
MORPHOLOGICAL VARIANTS OF SOFT PALATE AND ITS CORRELATION WITH TYPE OF MALOCCLUSION: A DIGITAL CEPHELOMETRIC STUDY
}

\section{Dr. BHAVNA BARTHUNIA ${ }^{1}$, Dr. POULOMI BHAKTA ${ }^{2}$, Dr. CHAUHAN BHUMIT ISHVARLAL ${ }^{3}$, Dr. NEHA KRISHNAKUMAR RAGHUWANSHI ${ }^{4}$, Dr. MOHD. IKRAM SHAIKH ${ }^{5}$}

\author{
${ }^{1}$ Prof. \& HOD, Dept. of Oral Medicine \& Radiology, Daswani Dental College \& \\ Research Centre,Ranpur, Kota, Rajasthan, India \\ ${ }^{2}$ Senior Lecturer, Dept. of Oral Medicine \& Radiology, Daswani Dental College \& \\ Research Centre,Ranpur, Kota, Rajasthan, India \\ ${ }^{3}$ MDS, Dept. of Oral Medicine \& Radiology, Daswani Dental College \& Research \\ Centre,Ranpur, Kota, Rajasthan, India \\ ${ }^{4,5}$ PG Student, Dept. of Oral Medicine \& Radiology, Daswani Dental College \& \\ Research Centre,Ranpur, Kota, Rajasthan, India
}

Corresponding Email: poulomibhakta@gmail.com

\section{ABSTRACT:}

Introduction: Soft palate as part of nasopharyngeal and oropharyngeal apparatus has an important role in phonation, deglutition and respiration. More so soft palate being a part of velophayrngeal apparatus has a major role in maintaining velophayrngeal competence. Pharyngeal depth (PD) can be used to determine the velopharyngeal function and is called the Need's ratio.

Materials And Methods: The study sample comprised randomly selected 300 healthy kota Patients with either of Angles class 1, class 2, class 3 malocclusion with age ranging from 18 to 48 years. Clinically,in all subjects, the type of malocclusion was examined and categorized according to Angle's classification of malocclusion. The morphological variants of soft palate were also assessed on digital lateral cephalogram and were allocated to one of the six patterns as described by You et al. and variation of malocclusion and soft palate morphology between gender groups was also assessed and Need's ratio was calculated for all the subjects by the division of PD by VL.

Results: The frequency of rat tail (26.3\%) type of soft palate was seen in highest proportion, whereas the frequency of distorted S-shape (7.7\%) was least in both the genders. Patients 


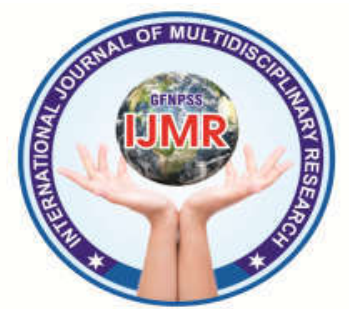

with Angle's class I malocclusion were most frequently found to have rat tail type soft palate, Angle's class II patients had leaf-shaped soft palate, and those with Angle's class III had crooked shaped soft palate. Velar length and velar width were significantly higher in male subjects than female subject.

Conclusion: The knowledge of morphological variants of the soft palate helps to get a better understanding of the velopharyngeal closure and also helps the clinician in successful functional and structural repair in cases of cleft palate and in the etiological study of OSAS, snoring, and other conditions.

Key words: Digital lateral cephalometry, Malocclusion, Need's ratio, Soft palate

INTRODUCTION:

The soft palate is a mobile flap suspended from the posterior border of the hard palate,sloping down and back between the oral and nasal parts of the pharynx. It is a thick fold of mucosa enclosing an aponeurosis, muscular tissue, vessels, nerves, lymphoid tissue, and mucus glands. ${ }^{1}$

The palate is formed by the fusion of three components, namely, two palatal processes and the primitive palate, which is formed by the frontonasal process. The intra-membranous ossification occurs in the mesoderm of palate to form the hard palate, whereas the soft palate is formed in the posterior region where the ossification does not occur. The most important muscle for velopharyngeal closure is the levator veli palatini (LVP). ${ }^{2}$

Normal palatine bones have horizontal shelves extending medially to fuse with one another. Here, they form a small posterior nasal spine associated with the insertion of paired uvulus muscles. ${ }^{3}$

The physical location of the palatine bones in space is directly related to the anteroposterior length of maxillary palatal shelves. Thus, foreshortening of the maxilla palate and/or the palatine bone will create conditions of velopharyngeal insufficiency even in the presence of a normal soft palate. ${ }^{4}$

Soft palate dysfunctions are frequently seen in cleft lip and palate patients, enlarged adenoids, obstructive sleep apnea, poorly retained maxillary denture and skeletal craniofacial malocclusion. $^{5}$

The velopharynx is a roughly rectangular space which is bordered anteriorly by velum (soft palate), posteriorly by posterior pharyngeal wall, 


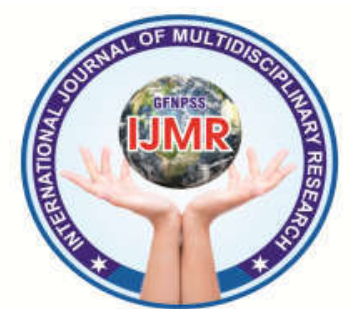

and laterally by right and left lateral pharyngeal walls. The contractions of these structures assist in closure of velopharyngeal port during the acts of eating, swallowing and speaking whereas, ${ }^{6}$

Radiographic techniques like lateral cephalography and computer tomography have been used for the study of morphological variations of soft palate. Studies on the dimensional analysis of soft palate and its surrounding structures have found variations in the morphology of soft palate. $^{7}$

Soft palate dysfunctions are most commonly observed in cleft palate patients who have short or otherwise abnormal velum, sub mucous cleft palate, excessively large tonsils or adenoids, webbing of the posterior tonsillar pillars, obstructive sleep apnea or skeletal craniofacial malocclusions. ${ }^{8}$

Patients presented with moderate to severe skeletal class II malocclusion with pre-existing enlarged adenoids or tonsils, allergies, asthma or obesity may develop obstructive sleep apnea (OSA) in future Knowledge about the difference in soft palate length and width, nasopharyngeal dimensions and Need's ratio in different skeletal malocclusions will assist in better understanding of the etiology of OSA syndrome. ${ }^{9}$

\section{MATERIALS \& METHODS:}

A total of 300 individuals, belonging to both gender between the age range of $18-48$ years were selected for the present study based in the selection criteria. The proposal for study was approved by the Institutional Ethical Committee. Their Digital Lateral cephalogram radiograph done using necessary radiation protection measures after taking their consent. The study population consist of 300 subjects belonging to both gender and all subjects were selected from Department of Oral Medicine and Radiology, Daswani dental college and research centre, ranpur, kota (RAJASTHAN) .

MATERIAL / EQUIPMENTS FOR THE STUDY:

- Plain mouth mirrors

- Single ended straight probe

- Explorers

- Tweezers

- Kidney tray

- Disposable gloves and mouth masks

FOR THE ASSESSMENT OF RADIOGRAPH 


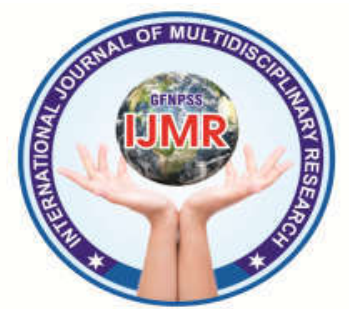

- Kodak $8000 \mathrm{C}$ with Kvp 72 and mA 10, employing Trophy DICOM software 6.1.2.0 required.

- Lead apron

The study will be conducted in the department of oral medicine and radiology, Daswani dental college and research centre, ranpur, kota (RAJASTHAN). The study sample comprised randomly selected 300 healthy individuals without any speech abnormality or any other syndromes or diseases with age ranging from 18 to 48 years.

300 study subjects will divided into 6 groups:

Group 1: 18-23 years

Group 2: 24-28 Years

Group 3: 29-33 Years

Group 4: 34-38 Years

Group 5: 39-43 Years

Group 6: 44-48 Years

\section{RESULTS:}

Table 1 showing distribution of patients according to gender with different malocclusions.

Table 2 showing distribution of patients according to age with different malocclusions and intergroup correlation.
Table 3 showing distribution of shapes of soft palate according the age groups and intergroup correlation.

Table 4 showing distribution of shapes of soft palate according to the gender and intergroup correlation.

Table 5 showing distribution of shapes of soft palate in patients with according to types of malocclusion and intergroup correlation.

Table 6 showing correlation of mean velar length, velar width, pharyngeal depth, and Need's ratio with age groups.Velar length was highest in patients with age group of $44-48$ years $(31.14 \mathrm{~mm}$ \pm 1.12 )

Table 7 showing correlation of mean velar length, velar width, pharyngeal depth, and Need's ratio with gender Velar length was higher in male subjects $(31.08 \mathrm{~mm} \pm 1.48)$ than female subject $(30.72 \mathrm{~mm} \pm 1.44)$.

Table 8 showing correlation of mean velar length, velar width, pharyngeal depth, and Need's ratio with types of soft palate.

Table 9 showing correlation of mean velar length, velar width, pharyngeal depth, and Need's ratio with types of malocclusion Velar length was highest in patients with class 2 malocclusion (30.96 $\mathrm{mm} \pm 1.02$ ) followed by class 3 


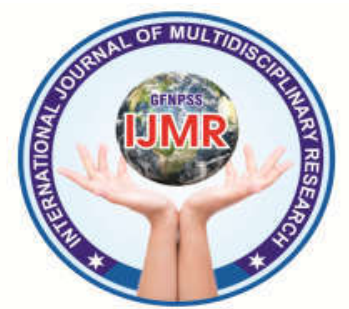

malocclusion $(30.93 \mathrm{~mm}= \pm 1.45)$ and less in patients with class 1 malocclusion (30.82 $\mathrm{mm} \pm 1.84)$.

\section{TABLES}

Table 1: Distribution of patients according to gender with different type of Angle's malocclusions:

\begin{tabular}{c|c|c|c}
\hline \multirow{2}{*}{ Tṛpe of Angle's malocclusion } & \multicolumn{3}{|c}{ Gender distribution } \\
\cline { 2 - 4 } & Male & Female & Total \\
\hline Class I malocclusion & $47(47 \%)$ & $53(53 \%)$ & 100 \\
\hline Class II malocclusion & $51(51 \%)$ & $49(49 \%)$ & 100 \\
\hline Class III malocclusion & $55(55 \%)$ & $45(45 \%)$ & 100 \\
\hline Total & $153(51 \%)$ & $147(49 \%)$ & 300 \\
\hline Chi Square Value & & 1.281 \\
\hline Pralue & \multicolumn{3}{|c}{0.527 (NS) } \\
\hline
\end{tabular}

\begin{tabular}{|c|c|c|c|c|}
\hline \multirow[b]{2}{*}{ Age group } & \multicolumn{4}{|c|}{ Type of Angle's malocclusion } \\
\hline & Class I & Class II & Class III & Total \\
\hline $\begin{array}{c}\text { Group } 1 \\
\text { (18-23 years) }\end{array}$ & $18(36.7 \%)$ & $17(34.7 \%)$ & $14(28.6 \%)$ & 49 \\
\hline $\begin{array}{c}\text { Group } 2 \\
\text { 24-28 Years) }\end{array}$ & $9(20.9 \%)$ & $15(34.9 \%)$ & $19(44.2 \%)$ & 43 \\
\hline $\begin{array}{c}\text { Group } 3 \\
\text { 29-33 Years) }\end{array}$ & $18(28.1 \%)$ & $21(32.8 \%)$ & $25(39.1 \%)$ & 64 \\
\hline $\begin{array}{c}\text { Group } 4 \\
\text { 34-38 Years) }\end{array}$ & $14(33.3 \%)$ & $13(31 \%)$ & $15(35.7 \%)$ & 42 \\
\hline $\begin{array}{c}\text { Group } 5 \\
\text { 39-43 Years) }\end{array}$ & $26(40.6 \%)$ & $19(29.7 \%)$ & $19(29.7 \%)$ & 64 \\
\hline $\begin{array}{c}\text { Group } 6 \\
\text { 44-48 Years) }\end{array}$ & $15(39.5 \%)$ & $15(39.5 \%)$ & $8(21.1 \%)$ & 38 \\
\hline TOTAL & $100(33.3 \%)$ & $100(33.3 \%)$ & $100(33.3 \%)$ & 300 \\
\hline i Square Value & \multicolumn{4}{|c|}{9.475} \\
\hline$P$ value & \multicolumn{4}{|c|}{0.488 (NS) } \\
\hline
\end{tabular}

[Test used: chi square, $\mathrm{S}=$ significant, $\mathrm{NS}=$ non significant, $\mathrm{P}$ value $\leq 0.05=\mathrm{S}, \mathrm{P}>$ $0.05=\mathrm{NS}]$

\section{Table 3: Distribution of shapes of soft} palate according to the age groups and intergroup correlation.

\begin{tabular}{|c|c|c|c|c|c|c|c|}
\hline \multirow[b]{2}{*}{ Age group } & \multicolumn{7}{|c|}{ Shape of soft palate } \\
\hline & $\begin{array}{c}\text { Type I } \\
\text { Leaf }\end{array}$ & $\begin{array}{l}\text { Type 2 } \\
\text { Rat tail }\end{array}$ & $\begin{array}{l}\text { Type } 3 \\
\text { Butt like }\end{array}$ & $\begin{array}{l}\text { Type 4 } \\
\text { Straight } \\
\text { line }\end{array}$ & $\begin{array}{c}\text { Type 5 } \\
\text { Distorted S }\end{array}$ & $\begin{array}{l}\text { Type } 6 \\
\text { Crooked }\end{array}$ & Total \\
\hline $\begin{array}{c}\text { Group } 1 \\
\text { (18-23 years) }\end{array}$ & $\begin{array}{c}7 \\
(14.3 \%)\end{array}$ & $\begin{array}{c}4 \\
(8.2 \%)\end{array}$ & $\begin{array}{c}9 \\
(18.4 \%)\end{array}$ & $\begin{array}{c}11 \\
(22.4 \%)\end{array}$ & $\begin{array}{c}3 \\
\text { (6.1\%) }\end{array}$ & $\begin{array}{c}15 \\
(30.6 \%)\end{array}$ & 49 \\
\hline $\begin{array}{c}\text { Group } 2 \\
\text { (24-28 years) }\end{array}$ & $\begin{array}{c}10 \\
(23.3 \%)\end{array}$ & $\begin{array}{c}10 \\
(23.3 \%)\end{array}$ & $\begin{array}{c}2 \\
(4.7 \%)\end{array}$ & $\begin{array}{c}6 \\
(14 \%)\end{array}$ & $\begin{array}{c}5 \\
(11.6 \%)\end{array}$ & $\begin{array}{c}10 \\
(23.3 \%)\end{array}$ & 43 \\
\hline $\begin{array}{c}\text { Group } 3 \\
\text { (29-33 years) }\end{array}$ & $\begin{array}{c}11 \\
(17.2 \%)\end{array}$ & $\begin{array}{c}20 \\
(31.2 \%)\end{array}$ & $\begin{array}{c}4 \\
(6.2 \%)\end{array}$ & $\begin{array}{c}16 \\
(25 \%)\end{array}$ & $\begin{array}{c}4 \\
(6.2 \%)\end{array}$ & $\begin{array}{c}9 \\
(14.1 \%)\end{array}$ & 64 \\
\hline $\begin{array}{c}\text { Group } 4 \\
\text { (34-38 years) }\end{array}$ & $\begin{array}{c}6 \\
(14.3 \%)\end{array}$ & $\begin{array}{c}11 \\
(26.2 \%)\end{array}$ & $\begin{array}{c}6 \\
(14.3 \%)\end{array}$ & $\begin{array}{c}4 \\
(9.5 \%)\end{array}$ & $\begin{array}{l}3 \\
(7.1 \%)\end{array}$ & $\begin{array}{c}12 \\
(28.6 \%)\end{array}$ & 42 \\
\hline $\begin{array}{c}\text { Group } 5 \\
\text { (39-43 years) }\end{array}$ & $\begin{array}{c}12 \\
(188 \%)\end{array}$ & $\begin{array}{c}22 \\
(34.4 \%)\end{array}$ & $\begin{array}{c}3 \\
(47 \%)\end{array}$ & $\begin{array}{c}12 \\
(18.8 \%)\end{array}$ & $\begin{array}{c}6 \\
(5.3 \%)\end{array}$ & $\begin{array}{c}9 \\
(15.8 \%)\end{array}$ & 64 \\
\hline $\begin{array}{c}\text { Group } 6 \\
\text { (44-48 years) }\end{array}$ & $\begin{array}{c}9 \\
(23.7 \%)\end{array}$ & $\begin{array}{c}12 \\
(31.6 \%)\end{array}$ & $\begin{array}{c}3 \\
(7.9 \%)\end{array}$ & $\begin{array}{c}6 \\
(15.8 \%)\end{array}$ & $\begin{array}{c}2 \\
5.3 \%)\end{array}$ & $\begin{array}{c}6 \\
(15.8 \%)\end{array}$ & 38 \\
\hline Total & $\begin{array}{c}55 \\
\text { (18.3\%) }\end{array}$ & $\begin{array}{c}79 \\
(263 \%)\end{array}$ & $\begin{array}{c}27 \\
(9 \%)\end{array}$ & $\begin{array}{c}55 \\
(18.3 \%)\end{array}$ & $\begin{array}{c}23 \\
(7.7 \%)\end{array}$ & $\begin{array}{c}61 \\
(20.3 \%)\end{array}$ & 300 \\
\hline $\begin{array}{l}\text { Chi Square } \\
\text { Value }\end{array}$ & \multicolumn{7}{|c|}{32.885} \\
\hline P value & \multicolumn{7}{|c|}{0.134 (NS) } \\
\hline
\end{tabular}

[Test used: chi square, $\mathrm{S}=$ significant, $\mathrm{NS}=$ non significant, $\mathrm{P}$ value $\leq 0.05=\mathrm{S}, \mathrm{P}>$ $0.05=\mathrm{NS}]$ 


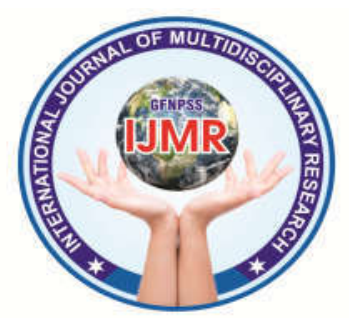

Table 4: Distribution of shapes of soft palate according to the gender and intergroup correlation.

\begin{tabular}{|c|c|c|c|c|c|c|c|}
\hline \multirow[b]{2}{*}{ Geader } & \multicolumn{7}{|c|}{ Shape of soft patate } \\
\hline & $\begin{array}{l}\text { Iype I } \\
\text { (Leaf) }\end{array}$ & $\begin{array}{l}\text { Iype } 2 \\
\text { (rat tail) }\end{array}$ & $\begin{array}{l}\text { Type } 3 \\
\text { (butt) }\end{array}$ & $\begin{array}{l}\text { Type 4 } \\
\text { (suraight } \\
\text { liae) }\end{array}$ & $\begin{array}{c}\text { Type 5 } \\
\text { (distorted s) }\end{array}$ & $\begin{array}{c}\text { Type 6 } \\
\text { (crooked) }\end{array}$ & $\begin{array}{c}\text { Tota } \\
1\end{array}$ \\
\hline Male & $\begin{array}{c}30 \\
(19.6 \%)\end{array}$ & $\begin{array}{c}41 \\
(26.859)\end{array}$ & $\begin{array}{c}12 \\
(78 \%)\end{array}$ & $\begin{array}{c}22 \\
(14.4 \%)\end{array}$ & $\begin{array}{c}11 \\
(82 \%)\end{array}$ & $\begin{array}{c}37 \\
(242 \%)\end{array}$ & 153 \\
\hline Female & $\begin{array}{c}25 \\
(17 \% 6)\end{array}$ & $\begin{array}{c}38 \\
(25990)\end{array}$ & $\begin{array}{c}15 \\
(10.26 \%)\end{array}$ & $\begin{array}{c}33 \\
(224 \% 6)\end{array}$ & $\begin{array}{l}12 \\
(8.2 \%)\end{array}$ & $\begin{array}{c}24 \\
(16.3 \%)\end{array}$ & 147 \\
\hline Total & $\begin{array}{c}55 \\
(183 \%)\end{array}$ & $\begin{array}{c}79 \\
(263 \%)\end{array}$ & $\begin{array}{l}27 \\
\text { (989) }\end{array}$ & $\begin{array}{c}55 \\
\text { (18.3\%) }\end{array}$ & $\begin{array}{c}23 \\
(0.7 \%)\end{array}$ & $\begin{array}{c}61 \\
(203 \%)\end{array}$ & 300 \\
\hline $\begin{array}{l}\text { Chi Square } \\
\text { Valae }\end{array}$ & \multicolumn{7}{|c|}{5798} \\
\hline P value & & & & 0.326 (NS) & & & \\
\hline
\end{tabular}

\begin{tabular}{|c|c|c|c|c|c|c|c|}
\hline \multirow[b]{2}{*}{ Variables } & \multicolumn{7}{|c|}{ AGE GROUPS } \\
\hline & $\begin{array}{c}\text { Group 1 } \\
\text { (18-23 } \\
\text { years) }\end{array}$ & $\begin{array}{l}\text { Group 2 } \\
\text { (24-28 } \\
\text { years) }\end{array}$ & $\begin{array}{c}\text { Group } 3 \\
\text { (29-33 } \\
\text { years) }\end{array}$ & $\begin{array}{c}\text { Group 4 } \\
\text { (34-38 } \\
\text { years) }\end{array}$ & $\begin{array}{c}\text { Group } 5 \\
\text { (39-43 } \\
\text { years) }\end{array}$ & $\begin{array}{c}\text { Group } 6 \\
\text { (44-48 } \\
\text { years) }\end{array}$ & $\begin{array}{c}P \\
\text { value }\end{array}$ \\
\hline $\begin{array}{c}\text { Velar } \\
\text { length } \\
(\mathrm{mm})\end{array}$ & $\begin{array}{l}30.45 \\
\pm 1.88\end{array}$ & $\begin{array}{l}31.04 \\
\pm 1.16\end{array}$ & $\begin{array}{l}30.95 \\
\pm 1.31\end{array}$ & $\begin{array}{l}31.13 \\
\pm 1.34\end{array}$ & $\begin{array}{l}30.82 \\
\pm 1.67\end{array}$ & $\begin{array}{l}31.14 \\
\neq 1.12\end{array}$ & 0.195 \\
\hline $\begin{array}{l}\text { Velar } \\
\text { width } \\
(\mathrm{mm})\end{array}$ & $\begin{array}{l}7,98 \\
\pm 1.69\end{array}$ & $\begin{aligned} & 8.18 \\
& \pm 1.57\end{aligned}$ & $\begin{aligned} & 7.76 \\
\pm & 1.60\end{aligned}$ & $\begin{array}{l}8.14 \\
=1.70\end{array}$ & $\begin{array}{l}7.83 \\
\pm 1.49\end{array}$ & $\begin{array}{l}7.89 \\
\pm 1.47\end{array}$ & 0.732 \\
\hline $\begin{array}{l}\text { haryngeal } \\
\text { depth } \\
(\mathbf{m m})\end{array}$ & $\begin{array}{l}26.28 \\
\pm 1.58\end{array}$ & $\begin{array}{l}26.99 \\
\pm 1.86\end{array}$ & $\begin{array}{l}27.14 \\
\pm 1.95\end{array}$ & $\begin{array}{l}26.68 \\
=1.81\end{array}$ & $\begin{array}{l}27.26 \\
\pm 1.97\end{array}$ & $\begin{array}{l}27.03 \\
\pm 1.75\end{array}$ & 0.082 \\
\hline eeds ratio & $\begin{array}{c}0.85 \\
\pm 0.05\end{array}$ & $\begin{array}{c}0.86 \\
\pm 0.05\end{array}$ & $\begin{array}{c}0.87 \\
\pm 0.06\end{array}$ & $\begin{array}{c}0.85 \\
\pm 0.05\end{array}$ & $\begin{array}{c}0.88 \\
\pm 0.05\end{array}$ & $\begin{array}{c}0.86 \\
\pm 0.05\end{array}$ & 0.035 \\
\hline
\end{tabular}

[Test used: One way ANOVA, $\mathrm{S}=$ [Test used: chi square, $\mathrm{S}=$ significant, $\mathrm{NS}=$ non significant, $\mathrm{P}$ value $\leq 0.05=\mathrm{S}, \mathrm{P}>$ $0.05=\mathrm{NS}]$

Table 5: Distribution of shapes of soft palate in patients according to types of Angle's malocclusion and intergroup correlation.

\begin{tabular}{|c|c|c|c|c|c|c|c|}
\hline \multirow[b]{2}{*}{$\begin{array}{c}\text { Type of } \\
\text { Angle's } \\
\text { Malocclusion }\end{array}$} & \multicolumn{7}{|c|}{ Shape of soft palate } \\
\hline & $\begin{array}{c}\text { Type 1 } \\
\text { Leaf } \\
\text { shaped }\end{array}$ & \begin{tabular}{|l} 
Type 2 \\
Rat tail
\end{tabular} & $\begin{array}{c}\text { Type } 3 \\
\text { Butt }\end{array}$ & $\begin{array}{c}\text { Type 4 } \\
\text { Straight } \\
\text { line }\end{array}$ & $\begin{array}{c}\text { Type 5 } \\
\text { Distorted s }\end{array}$ & $\begin{array}{c}\text { Type 6 } \\
\text { crooked }\end{array}$ & Total \\
\hline CLASS 1 & $\begin{array}{c}9 \\
(9 \%)\end{array}$ & $\begin{array}{c}45 \\
(45 \%)\end{array}$ & $\begin{array}{c}4 \\
(4 \%)\end{array}$ & $\begin{array}{c}20 \\
(20 \%)\end{array}$ & $\begin{array}{c}6 \\
(6 \%)\end{array}$ & \begin{tabular}{|c|}
16 \\
$(16 \%)$
\end{tabular} & 100 \\
\hline CLASS 2 & $\begin{array}{c}38 \\
(38 \%)\end{array}$ & $\begin{array}{c}24 \\
(24 \%)\end{array}$ & $\begin{array}{c}14 \\
(14 \%)\end{array}$ & $\begin{array}{c}11 \\
(11 \%)\end{array}$ & $\begin{array}{c}6 \\
(6 \%)\end{array}$ & $\begin{array}{c}7 \\
(7 \%)\end{array}$ & 100 \\
\hline CLASS 3 & $\begin{array}{c}8 \\
(8 \%)\end{array}$ & $\begin{array}{c}10 \\
(10 \%)\end{array}$ & $\begin{array}{c}9 \\
(9 \%)\end{array}$ & $\begin{array}{c}24 \\
(24 \%)\end{array}$ & $\begin{array}{c}11 \\
(11 \%)\end{array}$ & $\begin{array}{c}38 \\
(38 \%)\end{array}$ & 100 \\
\hline TOTAL & $\begin{array}{c}55 \\
(18.3 \%)\end{array}$ & $\begin{array}{c}79 \\
(26.3 \%)\end{array}$ & $\begin{array}{l}27 \\
(9 \%)\end{array}$ & $\begin{array}{c}55 \\
(18.3 \%)\end{array}$ & $\begin{array}{c}23 \\
(7.7 \%)\end{array}$ & $\begin{array}{c}61 \\
(20.3 \%)\end{array}$ & 300 \\
\hline $\begin{array}{c}\text { Chi Square } \\
\text { Value }\end{array}$ & & & & 92.825 & & & \\
\hline$P$ value & & & & $0.000(\mathrm{~S})$ & & & \\
\hline
\end{tabular}

[Test used: chi square, $\mathrm{S}=$ significant, $\mathrm{NS}=$ non significant, $\mathrm{P}$ value $\leq 0.05=\mathrm{S}, \mathrm{P}>$ $0.05=\mathrm{NS}]$

Table 6: Correlation of mean velar length, velar width, pharyngeal depth, and Need's ratio with age groups

significant, $\mathrm{NS}=$ non significant, $\mathrm{P}$ value $\leq$ $0.05=\mathrm{S}, \mathrm{P}>0.05=\mathrm{NS}]$

Table 7: Correlation of mean velar length, velar width, pharyngeal depth, and Need's ratio with gender.

\begin{tabular}{|c|c|c|c|c|c|}
\hline \multirow{2}{*}{ Variables } & \multicolumn{4}{|c|}{ GENDER } \\
\cline { 2 - 5 } & \multicolumn{2}{|c|}{ Male } & \multicolumn{2}{c|}{ Female } & \multirow{2}{*}{ P value } \\
\cline { 2 - 5 } & mean & Sd & Mean & Sd & \multirow{2}{*}{$0.036(\mathrm{~S})$} \\
\hline $\begin{array}{c}\text { Velar length } \\
\text { (mm) }\end{array}$ & 31.08 & 1.48 & 30.72 & 1.44 & 0.009 (S) \\
\hline $\begin{array}{c}\text { Velar width } \\
\text { (mm) }\end{array}$ & 8.17 & 1.43 & 7.70 & 1.69 & 0.613 (NS) \\
\hline $\begin{array}{c}\text { Pharygeal } \\
\text { depth (mm) }\end{array}$ & 26.98 & 1.81 & 26.87 & 1.91 & 0.125 (NS) \\
\hline Needs ratio & 0.86 & 0.05 & 0.87 & 0.05 & \\
\hline
\end{tabular}

[Test used: unpaired $\mathrm{T}$ test, $\mathrm{S}=$ significant, $\mathrm{NS}=$ non significant, $\mathrm{P}$ value $\leq 0.05=\mathrm{S}, \mathrm{P}$ $>0.05=\mathrm{NS}]$

Table 8: Correlation of mean velar length, velar width, pharyngeal depth, and Need's ratio with types of soft palate. 


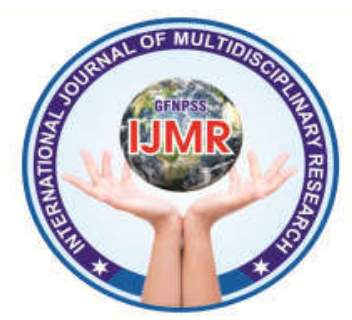

\begin{tabular}{|c|c|c|c|c|c|c|}
\hline \multirow[b]{2}{*}{ Variables } & \multicolumn{6}{|c|}{ TYPE OF SOFT PALATE } \\
\hline & $\begin{array}{l}\text { type } 1 \\
\text { (leaf) }\end{array}$ & $\begin{array}{l}\text { type } 2 \\
\text { (rat tail) }\end{array}$ & $\begin{array}{l}\text { type } 3 \\
\text { (butt) }\end{array}$ & $\begin{array}{l}\text { type } 4 \\
\text { (straight } \\
\text { line) }\end{array}$ & $\begin{array}{c}\text { type } 5 \\
\text { (distorted } \\
\text { s) }\end{array}$ & $\begin{array}{c}\text { type } 6 \\
\text { (crooked) }\end{array}$ \\
\hline $\begin{array}{l}\text { Velar length } \\
\text { (min) }\end{array}$ & $\begin{array}{c}30.59 \pm \\
1.00\end{array}$ & $\begin{array}{c}32.09 \pm \\
1.00\end{array}$ & $\begin{array}{c}30.68 \pm \\
1.43\end{array}$ & $\begin{array}{c}29.77 \pm \\
1.02\end{array}$ & $\begin{array}{c}30.24 \pm \\
0.85\end{array}$ & $\begin{array}{c}31.01 \pm \\
1.78\end{array}$ \\
\hline $\begin{array}{l}\text { Velar width } \\
\text { (mm) }\end{array}$ & $\begin{array}{c}8.86 \pm \\
0.75\end{array}$ & $\begin{array}{l}8.30 \pm \\
0.90\end{array}$ & $\begin{array}{l}9.18 \pm \\
0.82\end{array}$ & $\begin{array}{l}5.41 \pm \\
0.75\end{array}$ & $\begin{array}{c}6.30 \pm \\
0.39\end{array}$ & $\begin{array}{l}9.00 \pm \\
0.65\end{array}$ \\
\hline $\begin{array}{l}\text { Pharyngeal } \\
\text { depth }(\mathrm{mm})\end{array}$ & $\begin{array}{c}25.44= \\
0.70\end{array}$ & $\begin{array}{c}29.31 \pm \\
0.96\end{array}$ & $\begin{array}{c}24.84 \pm \\
0.82\end{array}$ & $\begin{array}{c}26.95 \pm \\
1.05\end{array}$ & $\begin{array}{c}27.67 \pm \\
0.91\end{array}$ & $\begin{array}{c}25.79= \\
0.97\end{array}$ \\
\hline Needs ratio & $\begin{array}{c}0.83 \pm \\
0.33\end{array}$ & $\begin{array}{l}0.91 \pm \\
0.02\end{array}$ & $\begin{array}{l}0.80 \pm \\
0.04\end{array}$ & $\begin{array}{c}0.89 \pm \\
0.03\end{array}$ & $\begin{array}{l}0.90 \pm \\
0.04\end{array}$ & $\begin{array}{l}0.82 \pm \\
0.03\end{array}$ \\
\hline
\end{tabular}

[Test used: One way ANOVA, $\mathrm{S}=$ significant, $\mathrm{NS}=$ non significant, $\mathrm{P}$ value $\leq$ $0.05=\mathrm{S}, \mathrm{P}>0.05=\mathrm{NS}]$

Table 9: Correlation of mean velar length, velar width, pharyngeal depth, and Need's ratio with Angle's malocclusion.

\begin{tabular}{|c|c|c|c|c|c|c|c|}
\hline \multirow{2}{*}{ Variables } & \multicolumn{6}{|c|}{ ANGLE'S MALOCCLUSION } & \multirow{2}{*}{$P$ value } \\
\hline & mean & sd & mean & sd & mean & sd & \\
\hline $\begin{array}{l}\text { Velar leugth } \\
(\mathrm{mm})\end{array}$ & 30.82 & 1.84 & 30.96 & 1.02 & 30.93 & 1.45 & 0.761 (NS) \\
\hline $\begin{array}{c}\text { Velar width } \\
\text { (mm) }\end{array}$ & 7.77 & 1.67 & 8.25 & 1.26 & 7.80 & 1.73 & $0.054(\mathrm{NS})$ \\
\hline $\begin{array}{l}\text { Pharyugeal } \\
\text { depth (mm) }\end{array}$ & 27.68 & 1.79 & 26.65 & 1.91 & 26.44 & 1.62 & $0.000(\mathrm{~S})$ \\
\hline Need's ratio & 0.89 & 0.05 & 0.86 & 0.05 & 0.85 & 0.05 & $0.000(\mathrm{~S})$ \\
\hline
\end{tabular}

[Test used: One way ANOVA, $\mathrm{S}=$ significant, $\mathrm{NS}=$ non significant, $\mathrm{P}$ value $\leq$ $0.05=\mathrm{S}, \mathrm{P}>0.05=\mathrm{NS}$ ]

\section{DISCUSSION:}

The lateral cephalogram is the most common diagnostic radiograph used in clinical orthodontics.
The important functions of soft palate, especially in maintaining the velopharyngeal closure and the dimensional changes of the soft palate and its surrounding structures, especially in the length and width, have been previously studied by some investigators Lateral cephalometric radiographs have been used since many years for evaluating soft palate.

\section{CONCLUSION:}

The morphology of the soft palate can be divided into six types according to their shape on lateral cephalometry. This

classification can help us better understand the diversity of the velar morphology in the median sagittal plane. These findings can be used as references for the research of velopharyngeal closure in cleft palate individuals and for etiological research of OSAS and other conditions. Radiographic analysis of morphological variations of soft palate may help in success of surgical procedures aimed for correcting deformities of soft palate. Very few studies available on correlation of morphological variation of soft palate and types of malocclusion. More studies are required with large sample size and detailed examination with 


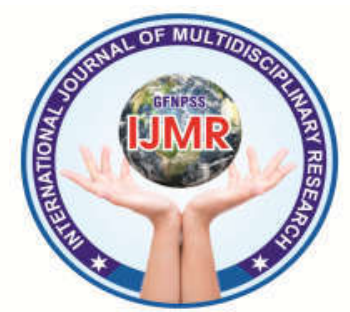

multicentre approach to establish this correlation.

In Different Sagittal and Vertical

\section{REFERENCES:}

1. Samdani D, Saigal A, Garg E. Correlation of morphological variants of soft palate and types of malocclusion: A digital lateral cephalometric study. J Indian Acad Oral Med Radiol 2015;27:366-71.

2. Michael H. Carstens, Pathologic anatomy of the soft palate, part 1: Embryology, the hard tissue platform, and evolution. J Cleft Lip Palate Cranio fac Anomal 2017;4:37-64.

3. Moore KL, Agur AM. Essential clinical anatomy. 2nd ed. Philadelphia: Lippincott Williams \& Wilkins; 2002.

4. Cohen SR, Chen L, Trotman CA, Burdi AR. Soft-palate myogenesis: A developmental field paradigm. Cleft Palate Craniofac J 1993;30:441-6.

5. Verma P, Verma KG, Kumaraswam KL, Basavaraju S, Sachdeva SK, Juneja S, et al. Correlation of morphological variants of the soft palate and need's ratio in normal individuals: A digital cephalometric study. Imaging Sci Dent 2014;44:1938.

6. Khoja A. Soft Palate Dimensions and Nasopharyngeal Depth (Need's Ratio)
Skeletal Patterns: A Lateral Cephalometric Study. Adv Dent \& Oral Health 2018;8(1):42-9.

7. Niu YM, Wang H, Zheng Q, He X, Zhang J, Li XM, et al. Morphology of the soft palate in normal humans with digital cephalometry. Hua Xi Kou Qiang Yi Xue Za Zhi 2006;24:321-2, 327.

8. Praveen $\mathrm{BN}$, Amrutesh $\mathrm{S}, \mathrm{Pal} \mathrm{S}$, Shubhasini AR, Vaseemuddin S. Various shapes of soft palate: a lateral cephalometric study. World J Dent 2011; 2: 207-10.

9. Khaitan $\mathrm{T}$, Pachigolla $\mathrm{R}$, Digital cephalometric analysis illustrating morphological variation of the soft palate. J Indian Acad Oral Med Radiol $2015 ; 27: 532-8$.

10. Standring S, editor. Gray's Anatomy. 40th ed. New York: Elsevier: Churchill Livingstone; 2006.

11. Carlson BM. Human Embryology and Developmental Biology. 5th ed. New York:Mosby/Elsevier; 2013.

12. Wada T, Satoh K, Tachimura T, Tatsuta U. Comparison of nasopharyngeal growth between patients with clefts and noncleft 


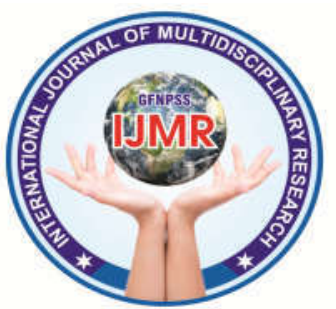

controls. Cleft Palate Craniofac J 1997;34:405-9.

13. Donnell F. Johns, Rod J. Rohrich, and Mariam Awada, Velopharyngeal Incompetence: A Guide for Clinical Evaluation American society of Plast reconstr surge.2003;112(7):35-9.

14. Johnston CD, Richardson A. Cephalometric changes in adult pharyngeal morphology.Eur J Orthod 1999;21:357 $\square 62$.

15. Guttal KS, Breh R, Bhat R, Burde KN, Naikmasur VG. Diverse morphologies of soft palate in normal individuals: A cephalometric perspective. J Indian Acad Oral Med Radiol 2012;24:15-9.

16. C. Vani, T. Vinila Lakshmi, V. Dheeraj Roy. Morphological variations of soft palate and influence of age on it: A digital cephalometric study. IAIM,2017; 4(10):72-76.

17. Pepin JL, Veale D, Ferretti GR, Mayer P, Levy PA. Obstructive sleep apnea syndrome:hooked appearance of the soft palate in awake patients cephalometric and CT findings.Radiology 1999; 210: 163-70. 